\title{
An Improved and Adaptive Approach in ANFIS to Predict Knee Diseases
}

\author{
Ranjit Kaur, Lovely Professional University, Phagwara, India \\ Kamaldeep Kaur, Lovely Professional University, Phagwara, India \\ Aditya Khamparia, Lovely Professional University, Phagwara, India \\ Divya Anand, Lovely Professional University, Phagwara, India
}

\begin{abstract}
Artificial intelligence is emerging as a persuasive tool in the field of medical science. This research work also primarily focuses on the development of a tool to automate the diagnosis of inflammatory diseases of the knee joint. The tool will also assist the physicians and medical practitioners for diagnosis. The diseases considered for this research under inflammatory category are osteoarthritis, rheumatoid arthritis and osteonecrosis. A five-layer adaptive neuro-fuzzy (ANFIS) architecture was used to model the system. The ANFIS system works by mapping input parameters to the input membership functions, input membership functions are mapped to the rules generated by the ANFIS model which are further mapped to the output membership function. A comparative performance analysis of fuzzy system and ANFIS system is also done and results generated shows that the ANFIS system outperformed fuzzy system in terms of testing accuracy, sensitivity and specificity.
\end{abstract}

\section{KEYWORDS}

Adaptive Neuro-Fuzzy, Fuzzy Systems, Osteoarthritis, Osteonecrosis, Predictive Models, Rheumatoid Arthritis

\section{INTRODUCTION}

Expert systems (ES) is a triumphant commercial product and enthralling research tool of artificial intelligence (AI) which transpired during early 1970s. ES is successfully employed in various fields like medical, agriculture, automatic control, data classification, decision analysis and airspace, specifically in medical field, where patient's health and safety measures are important. Knee is the hinge joint which plays vital role in skeletal system of human. The whole-body weight is on the knee joint. A number of categories of diseases could affect this vital part of the body such as inflammatory diseases, infectious diseases, neoplastic diseases, injuries, etc. The proposed system is designed to diagnose the inflammatory diseases of knee namely osteoarthritis, rheumatoid arthritis and osteonecrosis using adaptive neuro-fuzzy technique. 


\subsection{Orthopaedic Diseases}

With the advancement of the science, discovery of Roentgen's rays (x-rays) and discovery of bacteria by Louis Pasteur the new era of diseases of skeletal system emerged. From this various bones and joints diseases were came under orthopaedic, which are known as orthopaedic diseases (Pandey et al., 2009).

The human skeletal is made up of 206 bones where each bone of skeletal system could be covered under Inflammatory, Infective, and neoplastic disorder. Moreover, the human body is comprised of various joints as synovial fibrous, ball and socket joint and hinge joints (Moskowitz et al., 2006). Under the orthopaedic, there are spine, elbow, knee, ankle, wrist and shoulder body parts. Knee joint is the main weight bearing joint, so we have focus special attention towards those large hinge joint of the body. Knee comprises of three bones. The lower bone of thigh which is called Femur, upper part of the leg bone called Tibia and the knee cap bone is known as Patella as shown in Figure 1 (Chandna, 2018).

The main area of interest in knee are inflammatory pathology that is Osteoarthritis of knee, Rheumatoid arthritis of knee and osteonecrosis of knee and infective pathology like septic arthritis which can be acute septic arthritis, chronic septic arthritis and tuberculosis of the knee. Inflammatory diseases mean inflammation to the bony and soft tissue structures of the knee joint that causes inflammatory arthritis (Singh et al., 2012). Following diseases come under the inflammatory diseases:

- Osteoarthritis: Osteoarthritis is a common joint disorder (Justice et al., 2012). This is the progressive softening and disintegration of the articular cartilage. It is accompanied by new growth of cartilage and bone at the joint margin;

- Rheumatoid arthritis: Rheumatoid is an auto immune disease. It is the commonest cause of the chronic inflammatory disease. The most common characteristic features are elevated ESR, symmetrical polyarthritis and morning stiffness. Pathology of RA in knee, stage1 is synovitis and swelling in joint, stage 2 is early joint destruction with particular region and stage 3 is advanced joint destruction and deformity. This disease is common in all age group (children, young, old). It can be seropositive rheumatoid arthritis or seronegative rheumatoid arthritis. There are two outputs for the RA disease, one for adult and other for juvenile;

- Osteonecrosis: The avascular necrosis of the medial condyle of the femur is very common in the knee joint. It is often associated with the alcoholism and drug addictive. It is three times more common in females, above the age of 60 years.

Figure 1. Parts of knee

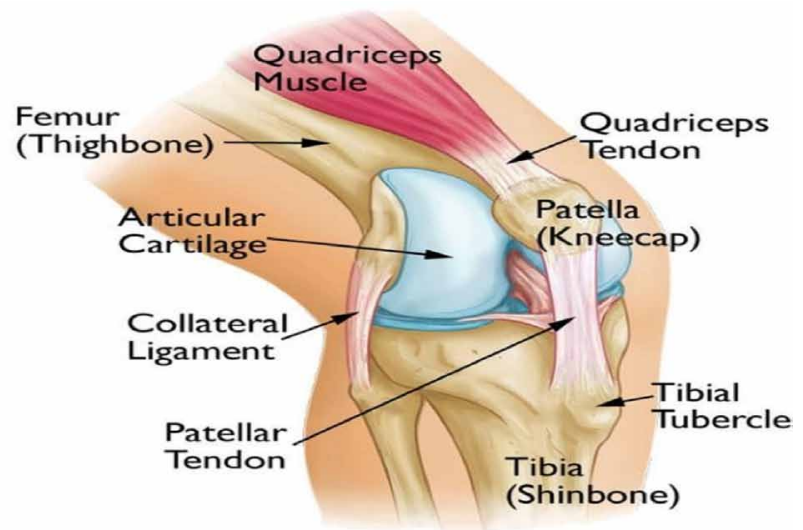




\section{LITERATURE REVIEW}

In 1970s, the first expert system developed for infectious blood diseases was MYCIN. It is a rule base system, implemented with 500 rules in LISP and deals with certain degree of belief using combining functions. With the advancement, many expert systems in various field is designed like DENDRAL, PROSPECTOR, ENT, cancer, dengue, gynecology, tumor, asthma and diabetes. Many Artificial Intelligence techniques like fuzzy logic, Artificial neural network, Genetic Algorithm, casebased reasoning, particle swarm optimization, rule-based reasoning is applied to design the expert systems. Literature study has proved that Artificial Neural Network (ANN) and Fuzzy logic (FL) has wide applicability in various fields like student modeling, medical, economics, traffic control, image processing, forecasting, manufacturing, electrical and electronic systems (Kar et al., 2014). ANN and FL methods are also widely applied in technical diagnostics and measurement (Viharos et al., 2015). In last few years, it is observed that ANFIS methods are used in various geotechnical engineering problems (Ali Cabalara et al., 2012).

An adaptive neuro-fuzzy system was developed to detect the abnormal red blood cells and it has given $96.6 \%$ accurate results. Inner and outer diameter of red blood cells were extracted using bounding box and adaptive local thresholding method after which the ANFIS method classifies the red blood cells as normal and abnormal (Khameneh et al., 2012). An application of ANFIS method is also seen to estimate the biochemical oxygen demand (BOD) of Surma River. Ten quality parameters were taken to estimate BOD and the performance of the ANFIS models was assessed through the mean squared error, correlation coefficient, Nash model efficiency and mean absolute error (Ahmed et al., 2017). ANFIS method was also used to predict the injection profiles during the development of oilfield. Two neuro-fuzzy systems based on grid partition and subtractive clustering was designed to compare the performance of both the systems. It is found in the research that grid partition based ANFIS systems outperforms subtractive clustering based ANFIS systems (Wei et al., 2007). A system was developed to predict the skin temperature in lower limb prostheses using adaptive neuro-fuzzy technique and Gaussian processes for machine learning. Results for both the techniques are measured and it is observed that both the techniques have comparable performance metrics (Mathur et al., 2016). In another application of ANFIS, a system was designed to predict thermal error compensation on CNC machine tools. For this research also two neuro-fuzzy systems based on grid partition and fuzzy c-means clustering was designed. It is found in the performance evaluation that fuzzy c-means clustering based ANFIS system is superior in terms of accuracy (Abdulshahed et al., 2015). ANFIS method is also applied for occupational risk assessment in the shipbuilding industry. Parameters taken for the study were type of incident, specialty, day and time, dangerous actions and dangerous situations involved in the incident. (Fragiadakis et al., 2014). An application in field of agriculture is also designed to predict the wheat grain yield on the basis of energy inputs. The system is also developed using ANN and the performance of ANN based system ANFIS systems are compared. The comparative results have shown that ANFIS system has predicted the yield more accurately and precisely than ANN systems (Khoshnevisan et al., 2014).

\section{KNOWLEDGE ACQUISITION}

The foremost step for designing the fuzzy system is to collect data and identify all input and output parameters. Knowledge acquisition is most problematical and strenuous tasks in the construction of expert systems. Sometime obstacles encountered while eliciting knowledge from human experts. These can be done by consulting with Orthopaedic expert doctor. The data is collected from the various sources such as research papers, books and expert doctors. Under the orthopaedic diseases, there are various categories of the knee joint. Major categories of the Knee joint are Inflammatory diseases, Infective Diseases and Neoplastic diseases as shown in Figure 2. The data is collected as 
shown in Figure 2. for the inflammatory category which covered osteoarthritis (OA), rheumatoid arthritis (RA) and osteonecrosis (ON) diseases.

\section{MATERIALS AND METHODS}

The proposed system is implemented using a five-layer adaptive neuro-fuzzy (ANFIS) architecture. ANFIS is an amalgamation of two famous soft computing techniques, i.e. Artificial Neural Network (ANN) and fuzzy logic. In contrast to Boolean logic which provides the answer in true or false, Fuzzy logic deals with uncertainty providing values ranging from 0 to 1 . Fuzzy logic has the ability to deal with the vague values; however, it doesn't have a specified method which could be used in the process of emulating human expertise into fuzzy rule base inference system. It also takes fairly a huge time for adjusting and deciding the membership functions. This drawback of fuzzy logic can be compensated by applying Artificial Neural Network (ANN) which can automatically decide and adjust the membership functions. This amalgamation of fuzzy logic and neural network will also help to reduce the error rates in formulating the fuzzy rules.

\subsection{Fuzzy Inference System}

A Fuzzy Inference System works in mainly three phases. Initially the crisp input values are fuzzified using membership functions which are decided using intuitionistic method. After the Fuzzification of input parameters, fuzzy rules in "IF-Then" format are designed by rigorous consultation with the expert. Inference engine is the heart of the fuzzy system which helps in reasoning and deducing the results based on the rules in the system. The inference engine generates an aggregated membership function through which a crisp output value is obtained by applying defuzzification methods like centroid. Figure 3 shows the detailed structure of the Fuzzy Inference System. There are several types of FIS, namely Takagi-Sugeno, Mamdani, and Tsukamoto. A Takagi-Sugeno FIS model was found to be widely used in the application of ANFIS method.

\subsection{Adaptive Network}

Figure 4 depicts an adaptive network which is an example of multiple layer feed forward neural network (Jang, 1993). Supervised learning algorithms are often used during the learning and training process of these networks. The adaptive networks are composed of a number of adaptive

Figure 2. Symptoms collected for the knee diseases

\begin{tabular}{|c|l|l|l|}
\hline \multirow{2}{*}{ \#Symptoms } & \multicolumn{3}{|c|}{ DISEASE NAME } \\
\cline { 2 - 4 } & Osteoarthritis & $\begin{array}{l}\text { Rheumatoid } \\
\text { Arthritis }\end{array}$ & 0steonecrosis \\
\hline 1. & Pain & Pain & Pain \\
\hline 2. & Age & ESR & Swelling \\
\hline 3. & Swelling & Age & Medial joint Tenderness \\
\hline 4. & Deformity & Bilateral Swelling & Restriction \\
\hline 5. & Restriction & Bilateral Tenderness & \\
\hline
\end{tabular}


Figure 3. Fuzzy inference system

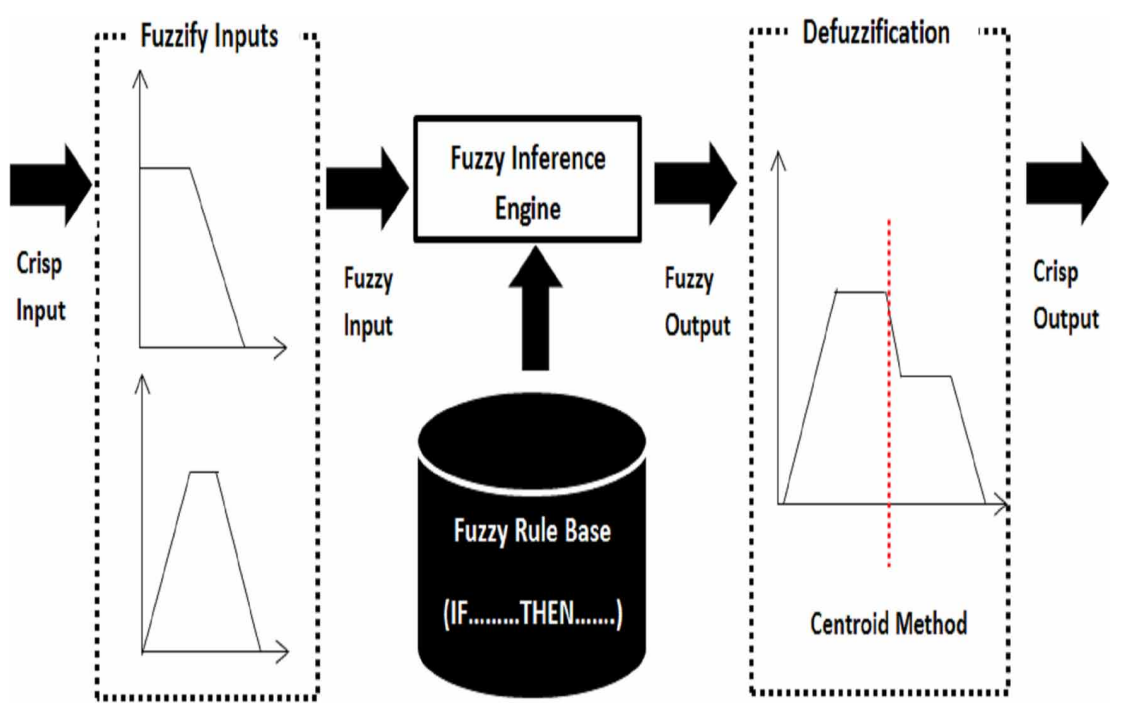

Figure 4. Adaptive network

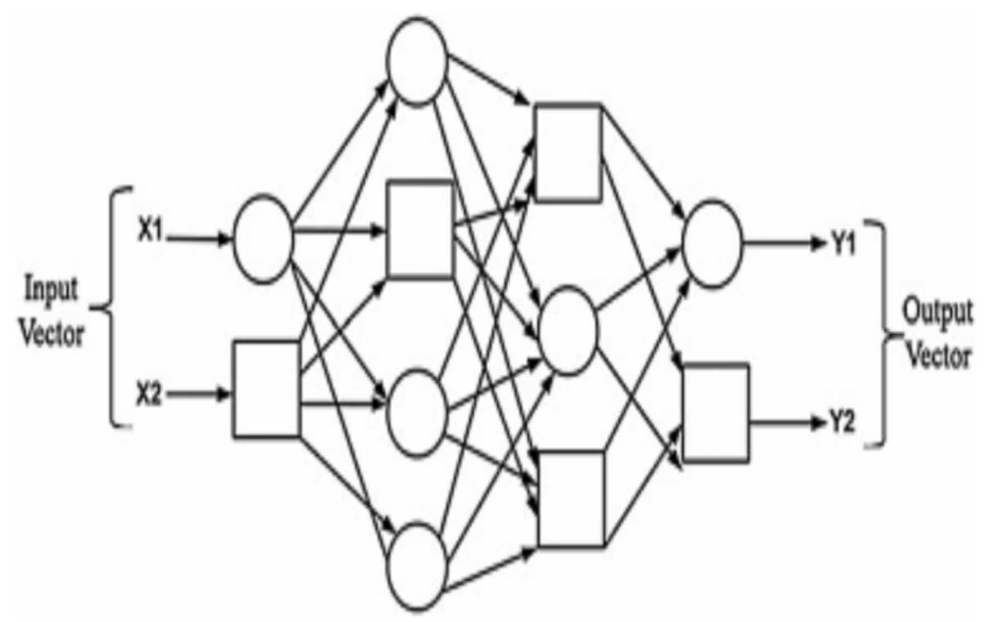

nodes which are directly interlinked with each other without having any value of weight between them. In the adaptive network every node has a specified function and job. The output of the nodes is dependent on the parameters and incoming signals which are available in the nodes. The rules generated after the training of the adaptive network will help to minimize the errors of adaptive network's output.

\subsection{ANFIS Architecture}

Adaptive Neuro-Fuzzy (ANFIS) architecture is an adaptive network which uses supervised learning. Takagi-Sugeno inference system is used to implement ANFIS method. Figure 5 shows the inference mechanism for Takagi-Sugeno FIS. Equation 1 and 2 defines two "IF-THEN" rules for Takagi-Sugeno model if it is assumed that there are two inputs a and $b$, and one output $f$ : 
ANTECEDENT

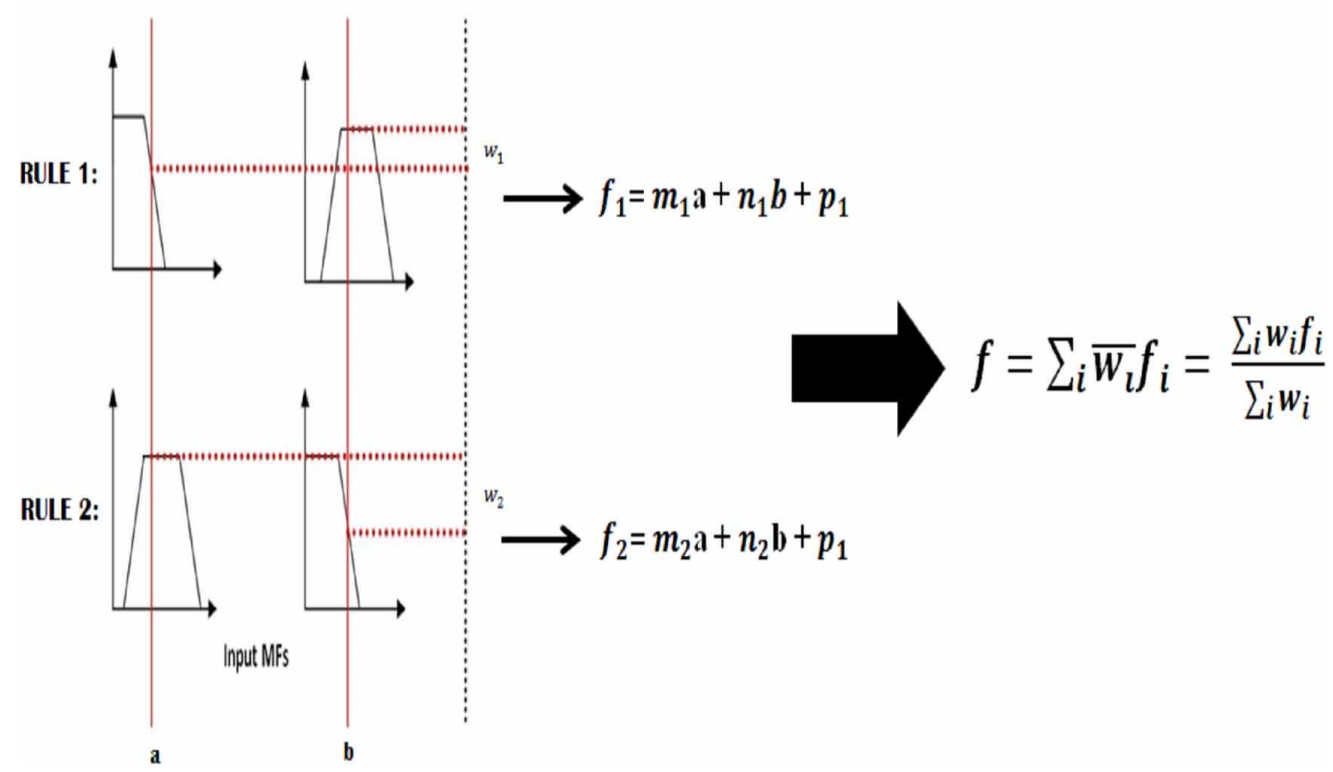

Rule 1: If $\mathrm{a}$ is $X_{1}$ and $\mathrm{b}$ is $Y_{1}$ Then $\mathrm{f} 1=m_{1} \mathrm{a}+n_{1} \mathrm{~b}+p_{1}$

Rule 2: If $\mathrm{a}$ is $X_{2}$ and $\mathrm{b}$ is $Y_{2}$ Then $\mathrm{f} 2=m_{2} \mathrm{a}+n_{2} \mathrm{~b}+p_{1}$

where $X_{1}, X_{2}$ and $Y_{1}, Y_{2}$ are the membership functions of each input a and $\mathrm{b}$ (part of the antecedent), while $m_{1}, n_{1}, p_{1}$ and $m_{2}, n_{2}, p_{2}$ are linear parameters in part-Then (consequent part) of TakagiSugeno fuzzy inference model (J.-S.R. Jang,1993).

Figure 6 shows the five-layer architecture of ANFIS model (Noureen, 2017). The first and fourth layers of the ANFIS architecture consists of adaptive node whereas the second, third and fifth layers consists of a fixed node. The description of all the five layers is given as follows:

Layer 1: The first layer of the ANFIS architecture has adaptive nodes. The inputs are mapped to the degree of membership value. The membership function on which the inputs are mapped can be a Gaussian membership function as defined in Equation 3, a bell membership function as defined in Equation 4, or any other type of membership function (Suparta et al., 2013):

$$
\begin{aligned}
\mu_{X i}(a) & =\exp \left[-\left(\frac{a-z_{i}}{2 x_{i}}\right)^{2}\right] \\
\mu_{X i}(a) & =\frac{1}{1+\left|\frac{a-z_{i}}{x_{i}}\right|^{2 y}}
\end{aligned}
$$




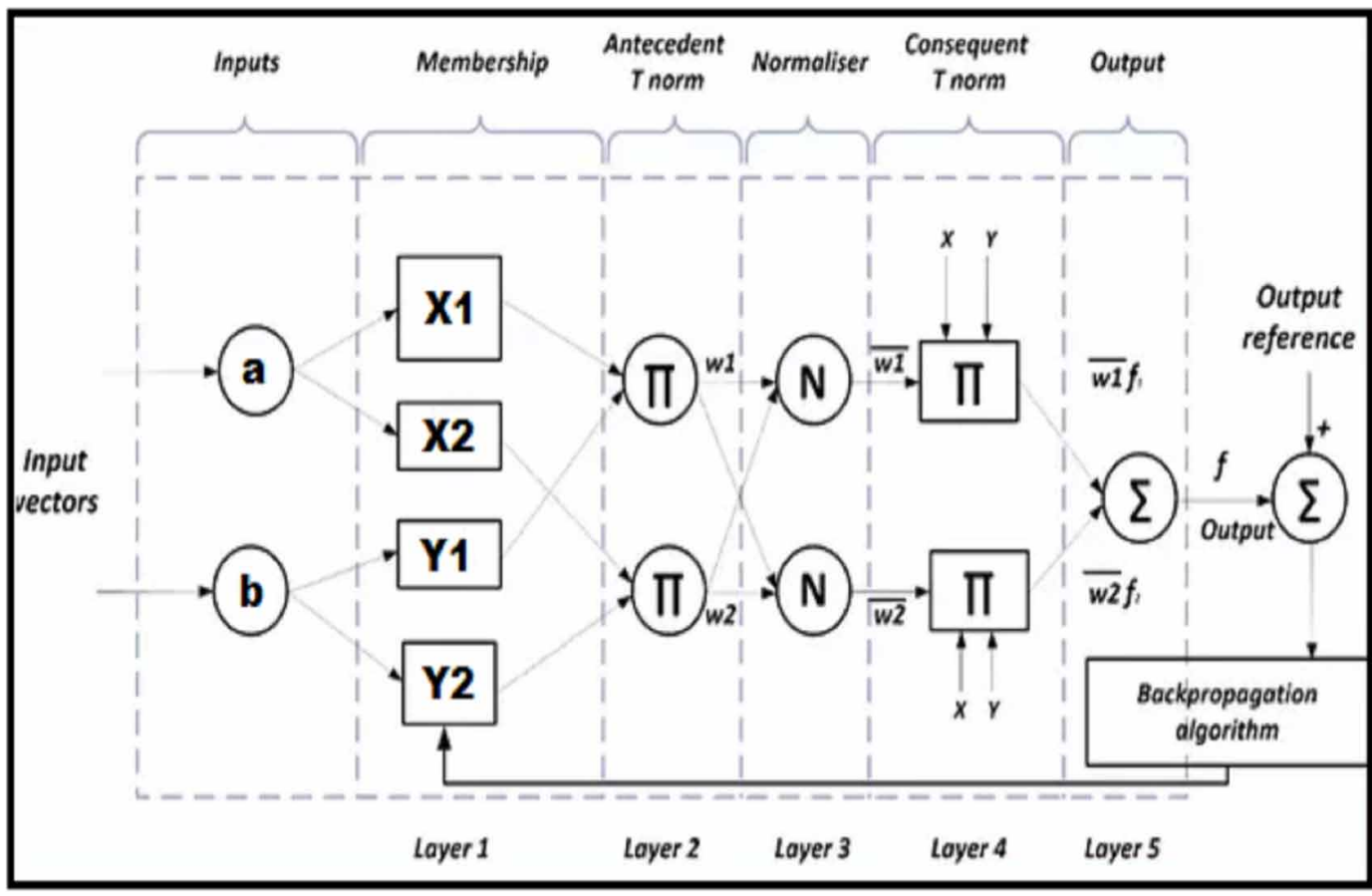

$O_{1, i}=\mu_{X i}(a), i=1,2$

$O_{1, i}=\mu_{Y i-2}(b), i=3,4$

where $\mu_{X i}$ and $\mu_{Y i-2}$ are the degree of membership functions for the fuzzy sets $X_{i}$ and $Y_{i}$ respectively. $a_{i}, b_{i}, c_{i}$ are the parameters of a membership function that can change the shape of the membership function.

Layer 2: In the second layer of ANFIS architecture every node is fixed or non-adaptive. Every node of this layer represents the firing strengths of the rules. The output from these nodes is generated by multiplying the incoming values to the node and outgoing values to the next node. In this layer, the T-norm operator such as AND operator is applied to get the output:

$O_{2 i}=w_{i}=\mu_{X i}(a) * \mu_{Y i}(b), \quad i=1,2$

where $w_{i}$ represents the firing strength of every rule.

Layer 3: In the third layer of ANFIS architecture every node is fixed or non-adaptive. Each node in this layer calculates the normalized firing strength of rule which is given as the ratio of firing strength of i-th rules to the total sum of firing strengths of all the rules: 
$O_{3 i}=\overline{w_{i}}=\frac{w_{i}}{\sum_{i} w_{i}}$

Layer 4: In the fourth layer of ANFIS architecture every node is an adaptive node which mapped to a node function defined as:

$$
O_{4 i}=\overline{w_{i}} f_{i}=\overline{w_{i}}\left(m_{i} a+n_{i} b+p_{i}\right)
$$

where $\overline{w_{i}}$ is the normalized firing strength of the rules and $m_{i} a, n_{i} b, p_{i}$ is a parameter in the node.

Layer 5: Fifth layer of ANFIS architecture contains a single node which is a fixed or non-adaptive node. This node calculates the final output as the sum of all the incoming values from the preceding nodes:

$$
O_{5 i}=\sum_{i} \bar{w}_{i} f_{i}=\frac{\sum_{i} w_{i} f_{i}}{\sum_{i} w_{i}}
$$

\subsection{Algorithm for Proposed System}

Figure 7 illustrates the algorithm designed for the proposed work which has been implemented using NeuroFuzzy Designer toolbox in MATLAB. The results obtained from the implementation of ANFIS model are then compared with the implementation of fuzzy model to evaluate the performance of both the models.

Figure 7. Algorithm for proposed system

STEP 1. Identification of parameters

STEP 2. Fuzzifying the input parameters using triangular and trapezoidal mf as defined in following equations:

$$
\begin{gathered}
\mu(x ; q, r, s, t)=\max \left(\min \left(\frac{x-q}{r-q}, 1 \frac{t-x}{t-s}\right), 0\right) \\
\quad \text { and } \\
\mu(x ; q, r, s)=\max \left(\min \left(\frac{x-q}{r-q}, \frac{s-x}{s-r}\right), 0\right)
\end{gathered}
$$

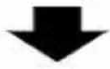

STEP 3. Designing Knowledge base in the form of "IF-THEN" rule.

Rule: If $\mathrm{x}$ is $\mathrm{A} 1$ and $\mathrm{y}$ is $\mathrm{B} 1$ Then $\mathrm{fl}=\mathrm{p} 1 \mathrm{x}+\mathrm{q} 1 \mathrm{x}+\mathrm{r} 1$

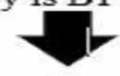

STEP 4. Training of FIS using Neural Networks

STEP 5. Performance Evaluation for training and testing data

STEP 6. Result Analysis for fuzzy and ANFIS 


\section{EXPERIMENTAL RESULTS}

The proposed model is implemented in MATLAB using Neuro-Fuzzy toolbox. Three types of inflammatory diseases of knee namely Osteoarthritis, Rheumatoid Arthritis, Osteonecrosis are predicted using ANFIS model. Figure 8 shows the five-layer ANFIS model for predicting Osteonecrosis disease. 4 input parameters are taken which are mapped to the input membership functions, input membership functions are mapped to the rules generated by the ANFIS model which are further mapped to the output membership function. Finally, the output membership functions are mapped to the single- valued output.

Data of 68 patients were taken to train the ANFIS model for predicting osteonecrosis disease. 232 patient's data were taken to train the ANFIS model for predicting Osteoarthritis and Rheumatoid Arthritis disease. Figure 9 shows the mapping of training data with the FIS output for osteonecrosis disease. After the training, the ANFIS system for predicting osteonecrosis disease is tested on 20 patients as shown in Figure 10.

Grid partition method is used to generate the FIS. While training of the system error tolerance was set to zero, number of Epochs were 10 and hybrid method for optimization was used. The ANFIS model generates 54 optimizes set of rules which are shown in Figure 11. Figure 12 displays the surface view of the ANFIS model where input 1 (pain) is mapped at $\mathrm{x}$-axis, input 2 (swelling) is mapped at $\mathrm{y}$-axis and the output (stage of osteonecrosis disease) is mapped at $\mathrm{z}$-axis.

The proposed system is implemented using fuzzy logic as well using neuro-fuzzy technique. Figure 13 illustrates the comparative results of the system being implemented using fuzzy and ANFIS on inflammatory diseases of the knee. The system for predicting Osteoarthritis disease is tested on 65 patients and the comparative results shows more accuracy, sensitivity and specificity obtained through ANFIS technique. 65 patient's data are used to test the system for predicting Rheumatoid Arthritis disease which also depicts a smaller number of false negative and false positive results obtained

Figure 8. ANFIS model structure

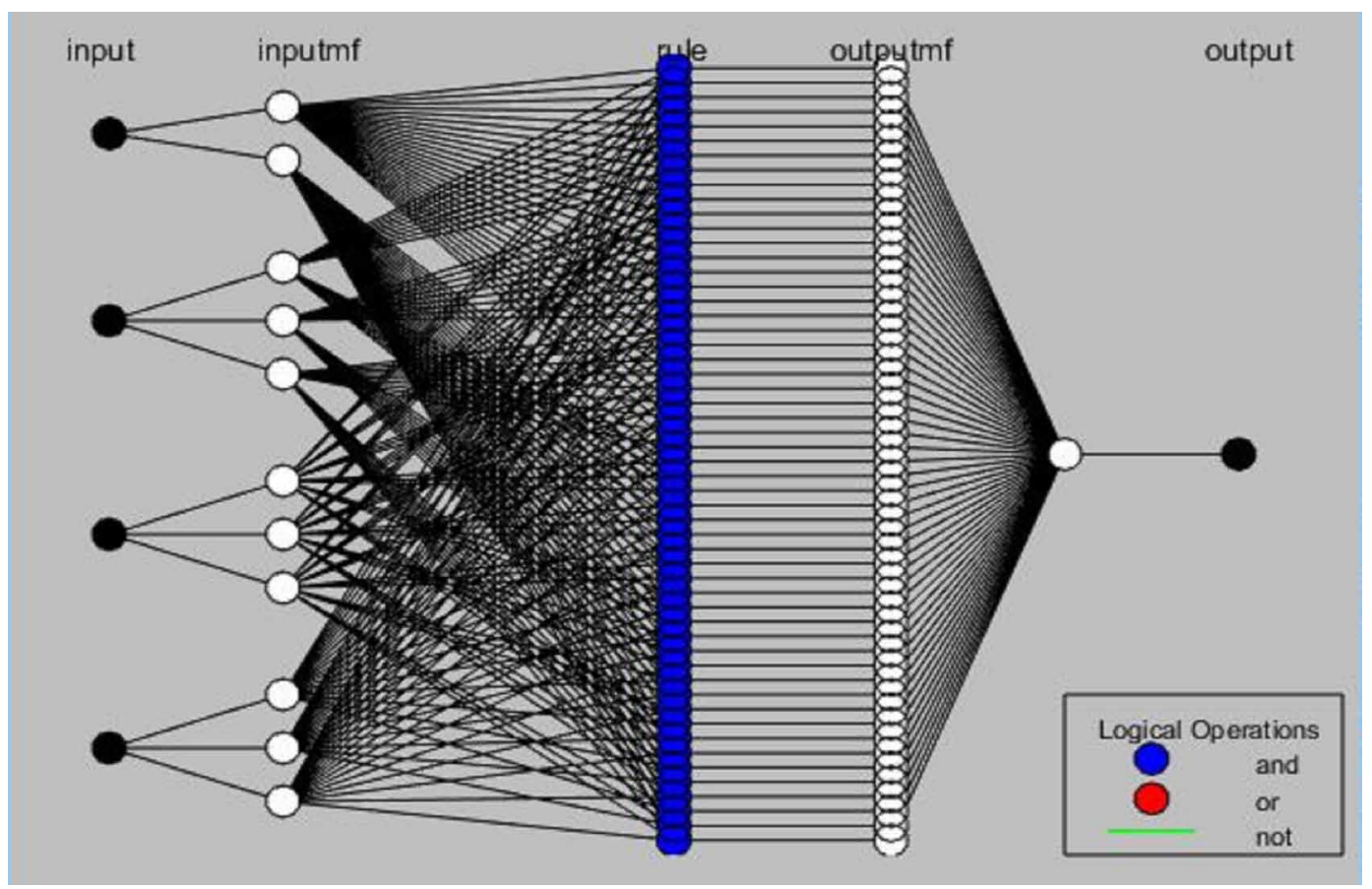


Figure 9. Mapping of training data and FIS output

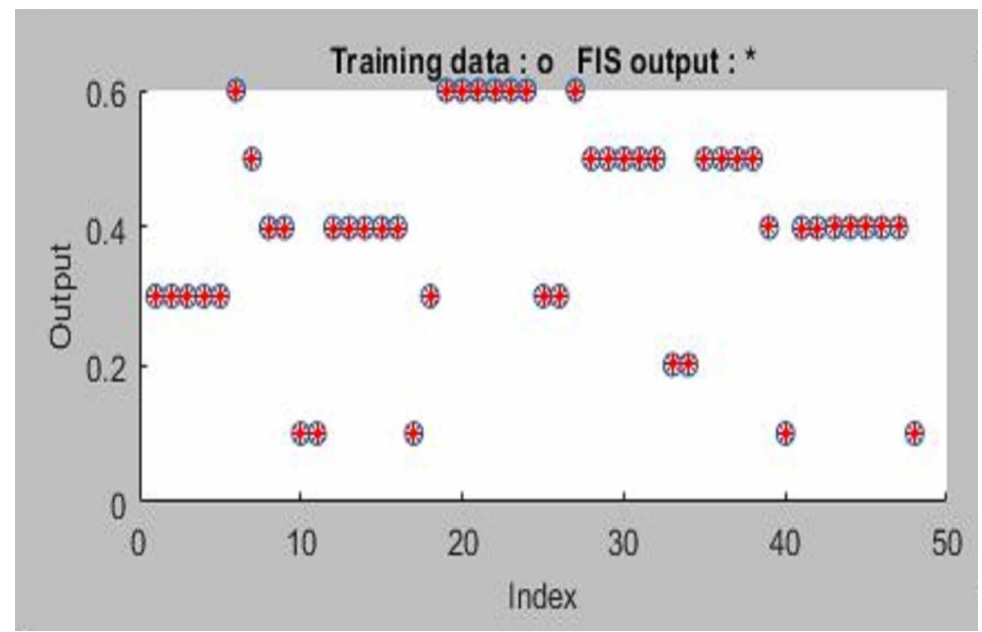

Figure 10. Mapping of testing data and FIS output

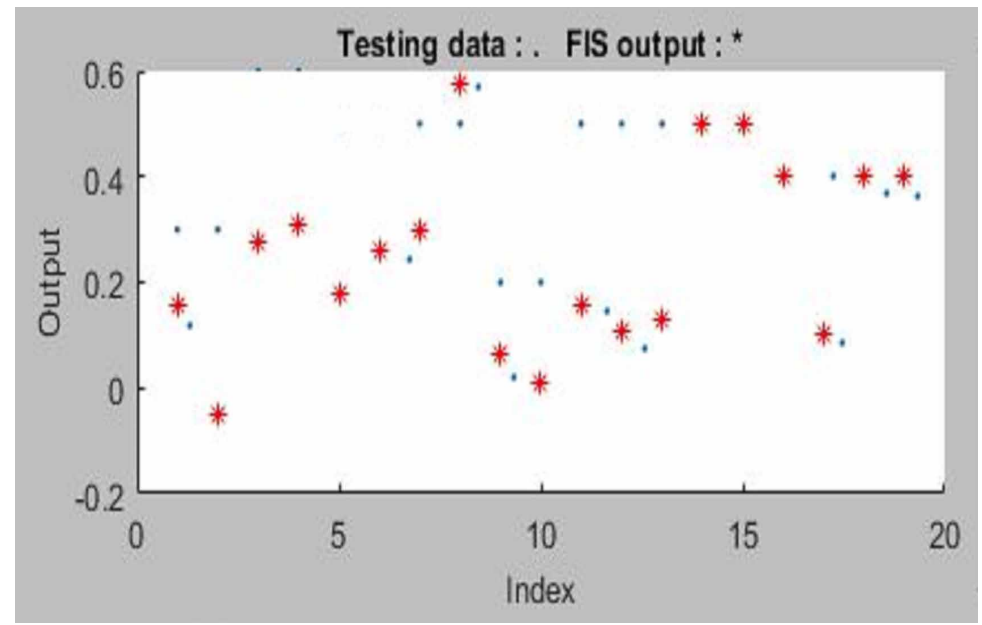

using ANFIS. For Osteonecrosis disease the system is tested on 40 patients and the ANFIS technique outperforms in this category as well. Figure 14, Figure 15 and Figure 16 presents the graphical view of the comparative results obtained after testing.

Mathematically, the testing accuracy, sensitivity, specificity can be calculated based on the number of True Positive (TP), True Negative (TN), False Positive (FP) and False Negative (FN) entities as defined by the following equations:

Positive Predictive value $(\mathrm{PPV})=\mathrm{TP} /(\mathrm{TP}+\mathrm{FP})$

Negative Predictive value $(\mathrm{NPV})=\mathrm{TN} /(\mathrm{TN}+\mathrm{FN})$

Sensitivity $=\mathrm{TP} /(\mathrm{TP}+\mathrm{FN}) * 100$ 


\section{Figure 11. Rule viewer}

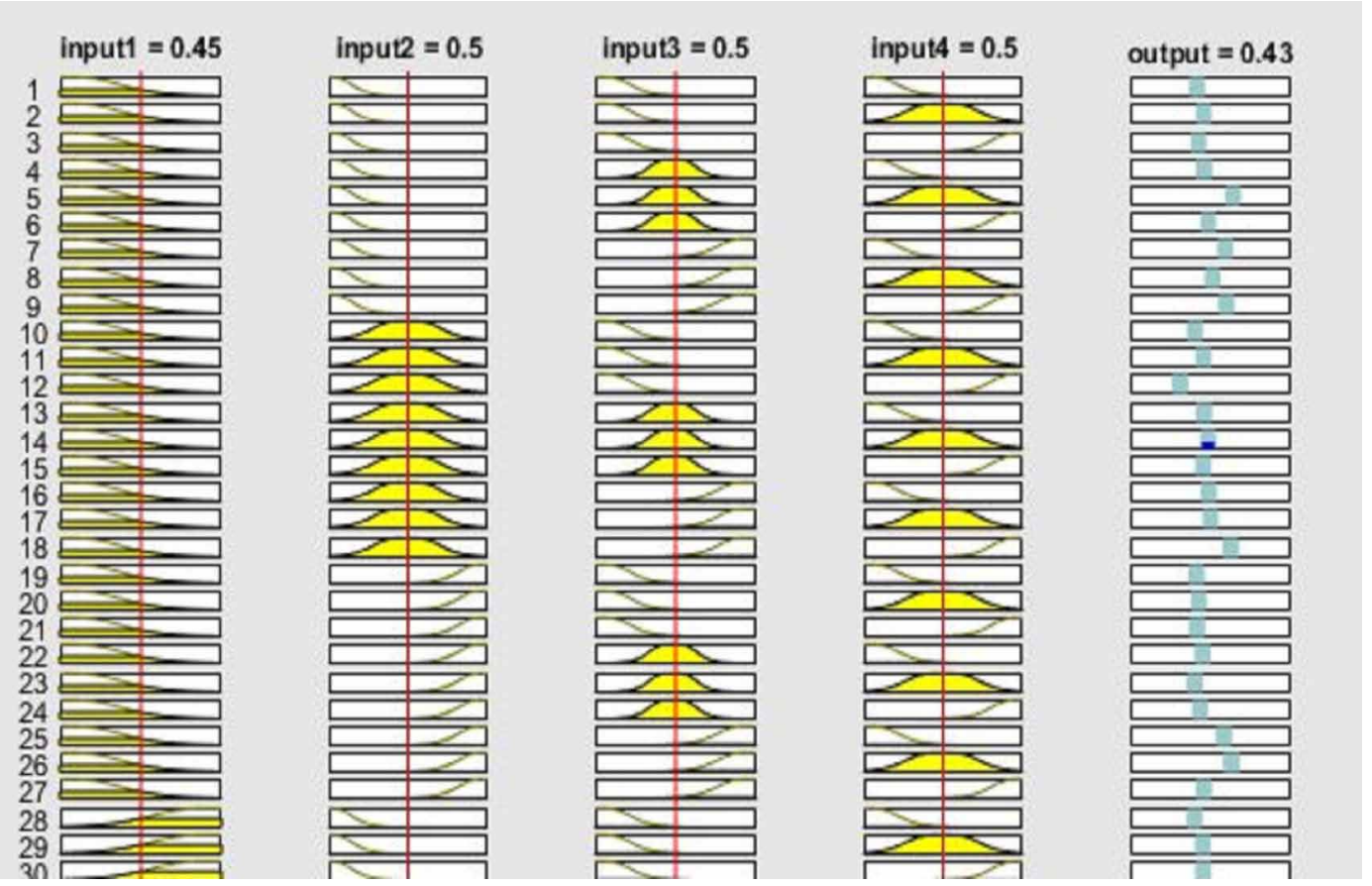

Figure 12. Surface viewer

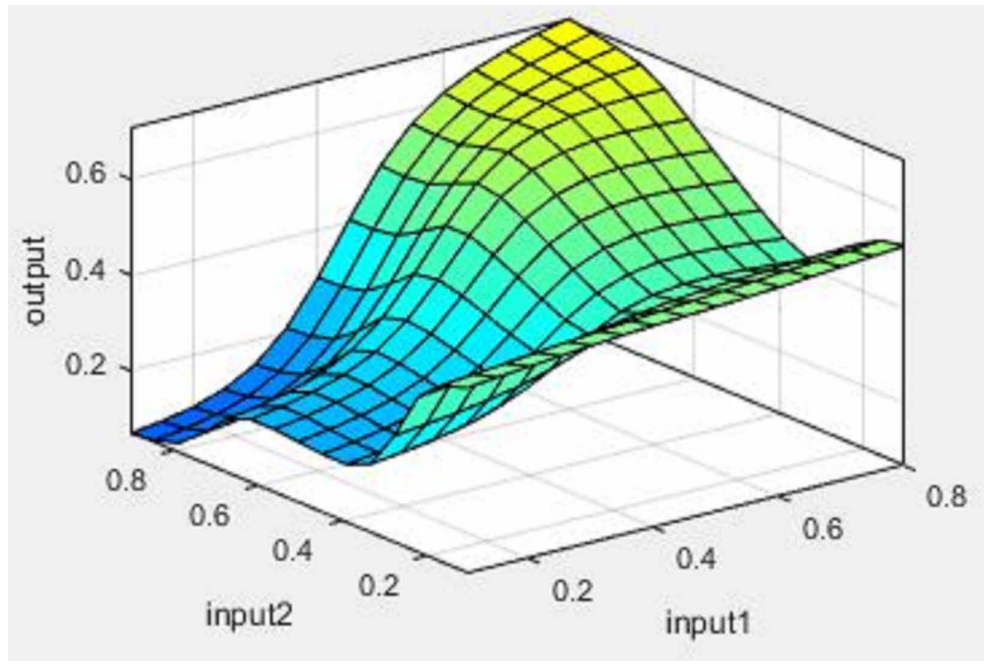

Specificity $=\mathrm{TN} /(\mathrm{TN}+\mathrm{FP}) * 100$

Testing Accuracy $=(\mathrm{TP}+\mathrm{TN}) /(\mathrm{TP}+\mathrm{FN}+\mathrm{TN}+\mathrm{FP}) * 100$

For instance, based on the above equations PPV, NPV, Sensitivity, Specificity and testing accuracy is calculated as follows for Osteoarthritis disease: 
Figure 13. Result analysis of Fuzzy and ANFIS system

\begin{tabular}{|c|c|c|c|c|c|c|c|c|c|}
\hline 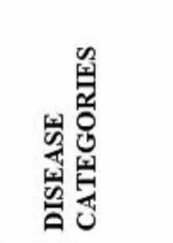 & 을 & 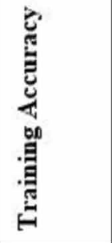 & 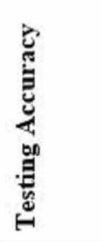 & 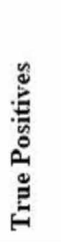 & 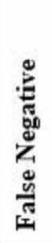 & 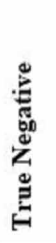 & 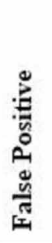 & 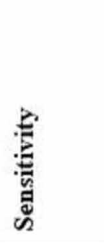 & 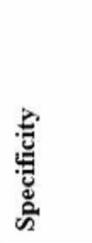 \\
\hline \multirow{2}{*}{ Osteoarthritis } & Fuzzy & - & $92.30 \%$ & 42 & 3 & 18 & 2 & $93.33 \%$ & $90.00 \%$ \\
\hline & ANFIS & $98.12 \%$ & $96.92 \%$ & 44 & 1 & 19 & 1 & 97.77 & $95.00 \%$ \\
\hline \multirow{2}{*}{$\begin{array}{c}\text { Rheumatoid } \\
\text { Arthritis }\end{array}$} & Fuzzy & - & $92.30 \%$ & 42 & 3 & 18 & 2 & $95.91 \%$ & $90.00 \%$ \\
\hline & ANFIS & $98.71 \%$ & $96.92 \%$ & 46 & 1 & 17 & 1 & 97.87 & $94.44 \%$ \\
\hline \multirow{2}{*}{ Osteonecrosis } & Fuzzy & - & $92.50 \%$ & 29 & 2 & 8 & 1 & $93.54 \%$ & $88.88 \%$ \\
\hline & ANFIS & $97.93 \%$ & $95.00 \%$ & 28 & 1 & 10 & 1 & $96.55 \%$ & $90.90 \%$ \\
\hline
\end{tabular}

Figure 14. Comparative results of ANFIS and Fuzzy model based on testing accuracy

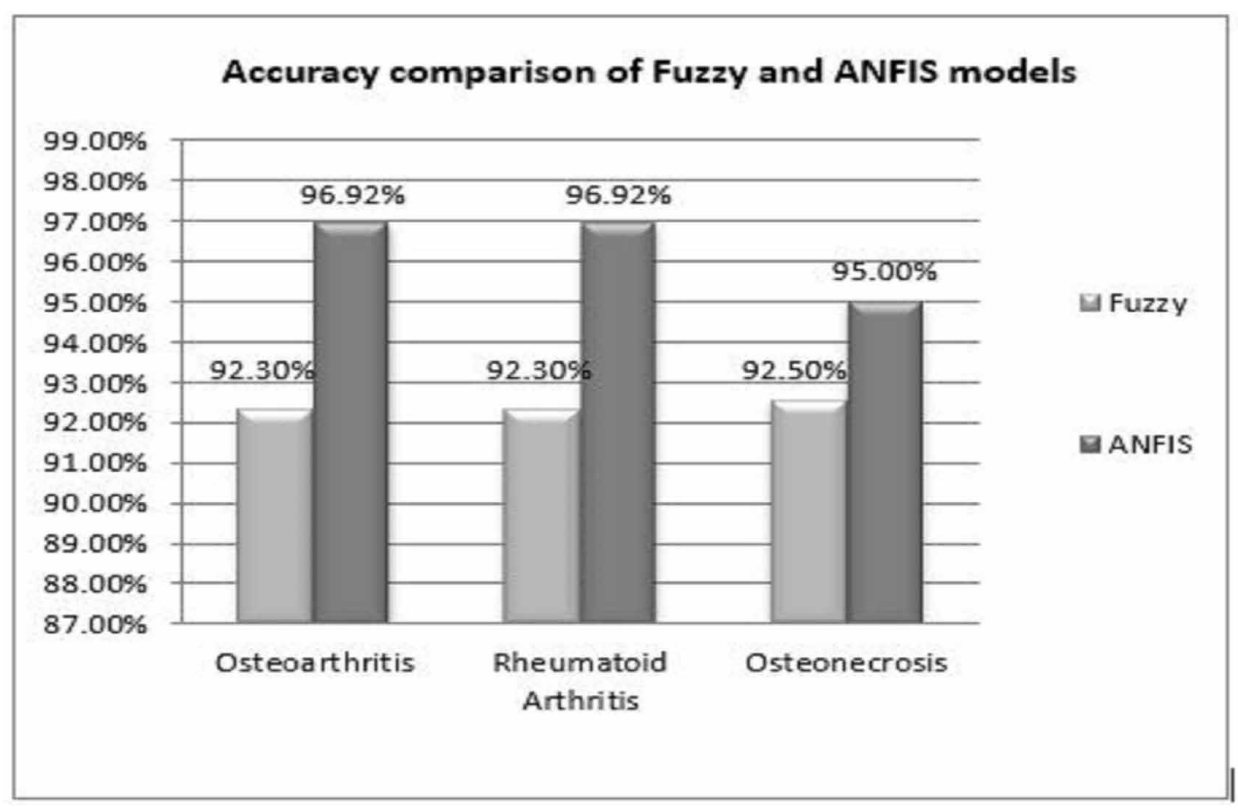

$\mathrm{PPV}=\mathrm{TP} /(\mathrm{TP}+\mathrm{FP})=44 /(44+1)=0.97$

$\mathrm{NPV}=\mathrm{TN} /(\mathrm{TN}+\mathrm{FN})=19 /(19+1)=0.95$

SENSITIVITY $=\mathrm{TP} /(\mathrm{TP}+\mathrm{FN})=44 /(44+1) * 100=97.77 \%$

$\mathrm{SPECIFICITY}=\mathrm{TN} /(\mathrm{TN}+\mathrm{FP})=19 /(19+1) * 100=95.00 \%$

TESTING ACCURACY $=(\mathrm{TP}+\mathrm{TN}) /(\mathrm{TP}+\mathrm{FN}+\mathrm{TN}+\mathrm{FP}) * 100=(44+19) /(44+1+19+1)$

$* 100=96.92 \%$ 
Figure 15. Comparative results of ANFIS and Fuzzy model based on sensitivity

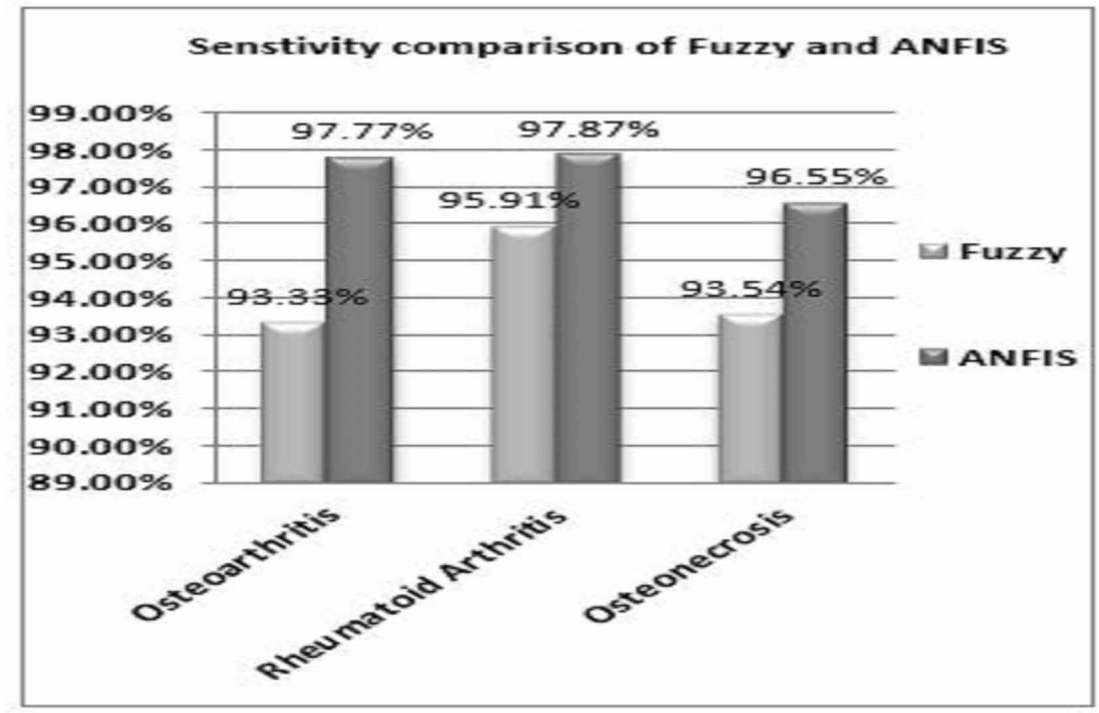

Figure 16. Comparative results of ANFIS and Fuzzy model based on specificity

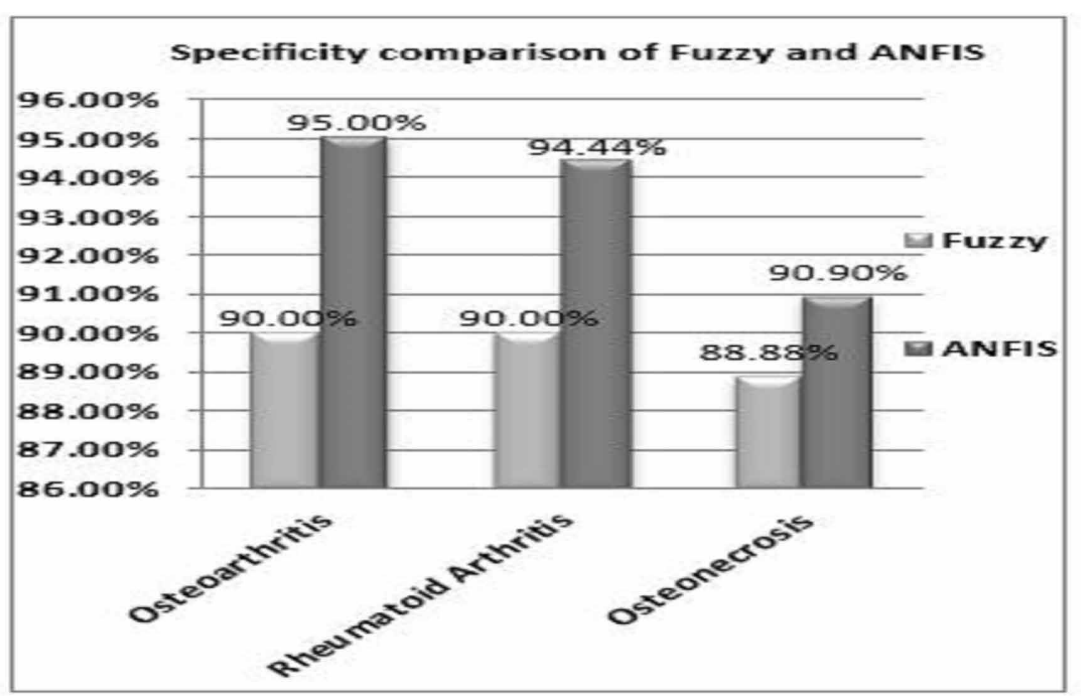

\section{DISCUSSION AND CONCLUSION}

The proposed system based on Adaptive Neuro-Fuzzy technique is designed to diagnose the inflammatory diseases of the knee. This fuzzy rule base technique is very helpful and accurate to diagnose the inflammatory diseases. Therefore, this proposed expert system could be used as a tool, which can abet the orthopaedic clinicians as well as learning system for orthopaedic medical students and practioners. Figures 14, 15 and 16 present a comparison among fuzzy and ANFIS model using accuracy, sensitivity and specificity respectively. Figure 14 illustrates that Osteoarthritis had $92.30 \%$ (fuzzy) and 96.92\% (ANFIS) accuracy, rheumatoid arthritis had 92.30\% (fuzzy) and 96.92\% (ANFIS) 
accuracy and osteonecrosis had 92.50\% (fuzzy) and 95\% (ANFIS) accuracy. Figure 15 depicts that osteoarthritis had 93.33\% (fuzzy) and 97.77\% (ANFIS) specificity, rheumatoid arthritis had $95.91 \%$ (fuzzy) and $97.87 \%$ (ANFIS) specificity and osteonecrosis had $93.54 \%$ (fuzzy) and $96.55 \%$ (ANFIS) specificity. Figure 16 illustrates that Osteoarthritis had 90\% (fuzzy) and 95\% (ANFIS) specificity, rheumatoid arthritis had 90\% (fuzzy) and $94.44 \%$ (ANFIS) specificity and osteonecrosis had $88.88 \%$ (fuzzy) and $90.90 \%$ (ANFIS) specificity. The obtained comparative results between fuzzy system and ANFIS system has unveiled that the ANFIS system has outperformed for all the diseases. Certainly, these fuzzification intelligent computing methods cannot remove the diagnose performed by medical practitioner but may acts as useful resource to assist patient after careful examination of their medical report. The proposed study is also an effort in that direction to propose an Artificial neural network and fuzzy based intelligent model for the efficient and effective diagnosis of knee related diseases.

This proposed research work can further be extended for another category like infective diseases and neoplastic diseases. The system could be designed using more parameters. Data of more patients could be collected to train the system. The system could be designed using other soft computing techniques like genetic algorithm, evolutionary computation and machine learning techniques. 


\section{REFERENCES}

Abdulshahed, A. M., Longstaff, A. P., \& Fletcher, S. (2015). The application of ANFIS prediction models for thermal error compensation on CNC machine tools. Applied Soft Computing, 27, 158-168.

Ahmed, A. M., \& Shah, S. M. A. (2017). Application of adaptive neuro-fuzzy inference system (ANFIS) to estimate the biochemical oxygen demand (BOD) of Surma River. Journal of King Saud University-Engineering Sciences, 29(3), 237-243.

Cabalara, A. F., Cevika, A., \& Gokceoglu, C. (2012). Some applications of Adaptive Neuro-Fuzzy Inference System (ANFIS) in geotechnical engineering. Computers and Geotechnics, 40, 14-33. doi:10.1016/j.compgeo.2011.09.008

Chandna, P., \& Chandra, M. (2018). Must read for knee replacement patients. Vaidam. Retrieved from https:// www.vaidam.com/knowledge-center/must-read-for-knee-replacement-patients

Fragiadakis, N. G., Tsoukalas, V. D., \& Papazoglou, V. J. (2014). An adaptive neuro-fuzzy inference system (anfis) model for assessing occupational risk in the shipbuilding industry. Safety Science, 63, 226-235. doi:10.1016/j.ssci.2013.11.013

Jang, J.-S. R. (1993). ANFIS: adaptive-network-based fuzzy inference system. IEEE Transactions on Systems, Man, and Cybernetics, 23(3), 665-685.

Justice, E. O., \& Taiwo, K. F. (2012). Fuzzy-based system for determining the severity level of knee osteoarthritis. International Journal of Intelligent Systems and Applications, 4(9), 46-53.

Kar, S., Das, S., \& Ghosh, P. K. (2014). Applications of neuro fuzzy systems: A brief review and future outline. Applied Soft Computing, 15, 243-259.

Khameneh, N. B., Arabalibeik, H., Salehian, P., \& Setayeshi, S. (2012). Abnormal red blood cells detection using adaptive neuro-fuzzy system. Studies in Health Technology and Informatics, 173, 30-34. PMID:22356952

Khoshnevisan, B., Rafiee, S., Omid, M., \& Mousazadeh, H. (2014). Development of an intelligent system based on ANFIS for predicting wheat grain yield on the basis of energy inputs. Information Processing in Agriculture, 1(1), 14-22. doi:10.1016/j.inpa.2014.04.001

Mathura, N., Gleska, I., \& Buisb, A. (2016). Comparison of adaptive neuro-fuzzy inference system (ANFIS) and Gaussian processes for machine learning (GPML) algorithms for the prediction of skin temperature in lower limb prostheses. Medical Engineering \& Physics, 38(10), 1083-1089. doi:10.1016/j.medengphy.2016.07.003

Moskowitz, R., Altman, R., \& Buckwalter, J. (2006). Osteoarthritis (4th ed.).

Noureen. (2017). How do I know about ANFIS. Quora. Retrieved from www.quora.com/How-do-I-knowabout-ANFIS

Pandey, S., \& Pandey, A. (2009). Clinical orthopaedic diagnosis. New Delhi: Jaypee Brothers Medical Publishers. doi: $10.5005 / \mathrm{jp} / \mathrm{books} / 11786$

Singh, A., \& Pandey, B. (2014). Intelligent techniques and applications in liver disorders: A survey. International Journal of Biomedical Engineering and Technology, 16(1), 27-70. doi:10.1504/IJBET.2014.065638

Singh, S., Kumar, A., Panneerselvam, K., \& Vennila, J. J. (2012). Diagnosis of arthritis through fuzzy inference system. Journal of Medical Systems, 36(3), 1459-1468. doi:10.1007/s10916-010-9606-9 PMID:20927572

Suparta, W., \& Alhasa, K. M. (2013, March). Estimation of precipitable water vapor using an adaptive neurofuzzy inference system technique. In Information and Communication Technology-EurAsia Conference (pp. 214-222). Springer; . doi:10.1007/978-3-642-36818-9_22

Takkar, S., Singh, A., \& Pandey, B. (2017). Application of machine learning algorithms to a well defined clinical problem: Liver disease. International Journal of E-Health and Medical Communications, 8(4), 38-60. doi:10.4018/IJEHMC.2017100103

Viharos, Zs. J., \& Kis, K. B. (2015). Survey on Neuro-Fuzzy systems and their applications in technical diagnostics and measurement. Measurement, 67, 126-136. doi:10.1016/j.measurement.2015.02.001

Wei, M., Bai, B., Sung, A. H., Liu, Q., Wang, J., \& Cather, M. E. (2007). Predicting injection profiles using ANFIS. Information Sciences, 177(20), 4445-4461. 
Ranjit Kaur is working as an Assistant Professor in the Department of Computer Science and Engineering at Lovely Professional University, Punjab, India. She has about two years of teaching experience and her research interests are machine learning, expert systems, and neural networks.

Kamaldeep Kaur is working as an Assistant Professor in the Department of Computer Science and Engineering at Lovely Professional University, Punjab, India. She has about two years of teaching experience and her research interests are data mining and machine learning.

Aditya Khamparia is working as an Assistant Professor in the Department of Computer Science and Engineering at Lovely Professional University, Punjab, India. He has about five years of teaching experience and his research interests are machine learning, e-learning, cognitive science and artificial thinking, soft computing, neural networks, and data mining.

Divya Anand is pursuing her PhD in the Department of Computer Science and Engineering at Lovely Professional University, Punjab, India. She has about five years of teaching experience and her research interests are machine learning, bioinformatics, and data mining. 